\title{
A rare case of abdominal adenoid basal cell carcinoma in a patient with a history of radiation therapy
}

\author{
Ji Hun Kim, Sun Eung Kim, Young Woo Cheon \\ Department of Plastic and Reconstructive Surgery, Gil Medical Center, Gachon University College of Medicine, Incheon, Korea
}

Basal cell carcinoma (BCC) is the most common skin cancer and its incidence is steadily increasing. Prior radiation therapy is one of the most important risk factors for BCC. Although the mechanism remains undefined, long-term studies have shown that people exposed to radiation have an increased risk of BCC. Despite the fact that BCC occurs most frequently in sun-exposed areas of the body, patients with a history of radiation therapy have an increased risk of BCC in areas previously exposed to radiation. Here, we report a case of adenoid BCC on the abdomen in a 67-year-old woman after radiation therapy post-hysterectomy.

Keywords Basal cell carcinoma / Radiotherapy / Skin neoplasm / Abdomen

\author{
Correspondence: Young Woo Cheon \\ Department of Plastic and \\ Reconstructive Surgery, Gil Medical \\ Center, Gachon University College of \\ Medicine, 21 Namdong-daero \\ 774beon-gil, Namdong-gu, Incheon \\ 21565, Korea \\ Tel: $+82-32-460-2770$ \\ Fax: +82-32-461-2774 \\ E-mail: youngwooc@gmail.com
}

Received: August 9, 2019• Revised: September 30, $2019 \bullet$ Accepted: October 22, 2019

pISSN: 2234-6163 • elSSN: 2234-6171 • https://doi.org/10.5999/aps.2019.01081 • Arch Plast Surg 2020;47:78-82

\section{INTRODUCTION}

Basal cell carcinoma (BCC) is the most common skin cancer, with over 2,000,000 cases diagnosed annually [1]. BCCs have three well-recognized growth patterns: nodular, superficial, and morphemic $[1,2]$. Histopathological variants of nodular BCCs include the solid (primordial), keratotic (pilar), cystic, and adenoid types. Adenoid BCC (ABCC) is a rare histopathological variant with a reported incidence of seven out of $103 \mathrm{BCC}$ cases [1-3]. ABCC can appear as pigmented or nonpigmented nodules or ulcers without a predilection for any particular site $[4,5]$. Because the major risk factor for $\mathrm{BCC}$ is exposure to ultraviolet $A$ and $B$ radiation, $B C C$ occurs most commonly in sun-exposed areas such as the face and neck ( $80 \%-90 \%)$, with the remaining $10 \%-15 \%$ of cases occurring in other areas, such as the abdomen, armpit, and chest $[4,6]$. However, in patients with a history of radiation treatment, the prevalence of $\mathrm{BCC}$ is higher than in patients with no history of radiation treatment [7-9]. Moreover, several reports have characterized radiogenic $\mathrm{BCC}$ as gen- erally more aggressive, difficult to excise completely, and more likely to recur than non-radiogenic BCC $[9,10]$. Therefore, it is important to conduct a close physical examination, especially of areas previously exposed to radiation, in patients with a history of radiation treatment [7-9,11]. Here, we report a case of $A B C C$ that showed unique histological and morphological features in a patient with a history of radiation therapy.

\section{CASE}

A 67-year-old Asian woman presented with a lesion in the abdominal area, which first appeared 5 years prior as a small protruding pigmented mass. The patient stated that the lesion initially appeared as a pigmented mass of less than $1 \mathrm{~cm}$ in diameter near the navel and grew slowly over 5 years. When she presented to the clinic, the lesion was $10.5 \times 7.1 \times 3.4 \mathrm{~cm}$, with a firm papillary shape and a brownish-black crust; additionally, the lesion was painless and pedunculated (Fig. 1). The patient had a history of leiomyosarcoma, for which she underwent a 


\section{Fig. 1. A clinical photo of the patient}

A localized, firm, and tender brownish-black papillary mass in the abdomen measuring $10.5 \times 7.1 \times 3.4 \mathrm{~cm}$.

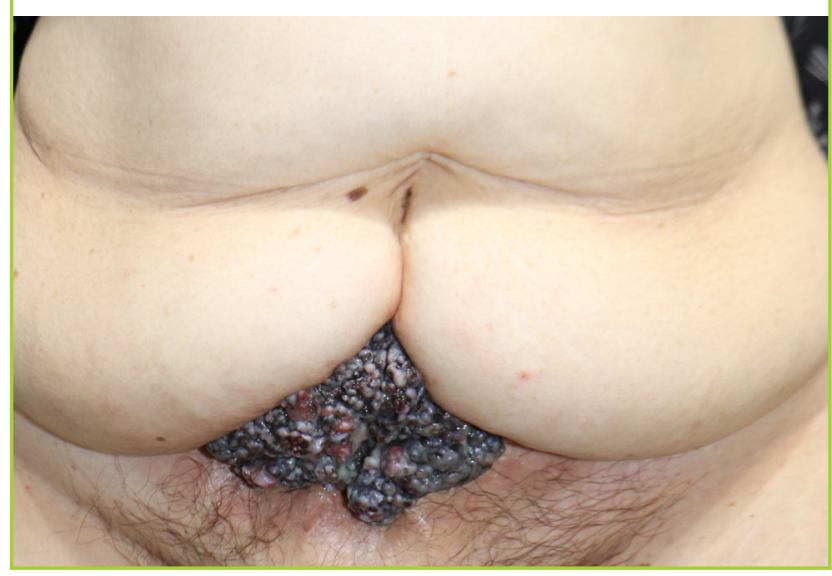

\section{Fig. 3. The resection specimen after wide excision}

Wide excision specimen of the adenoid basal cell carcinoma at surgery.

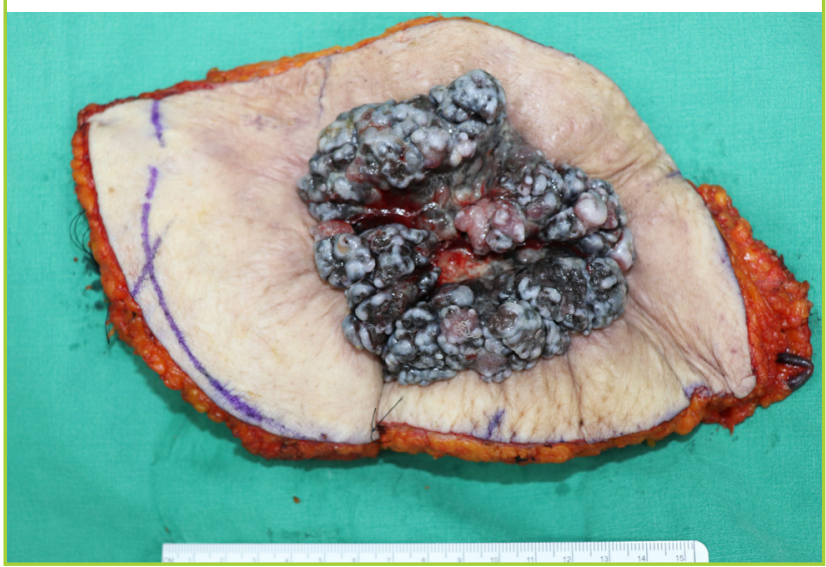

\section{Fig. 2. Assessment of the lesion on MRI}

(A) An irregular-shaped, exophytic soft tissue mass was observed at the level of the skin and the superficial subcutaneous layer of the lower abdominal wall. (B) Superficial ulcerative changes and intense enhancement were observed at the site of the lesion. MRI, magnetic resonance imaging.
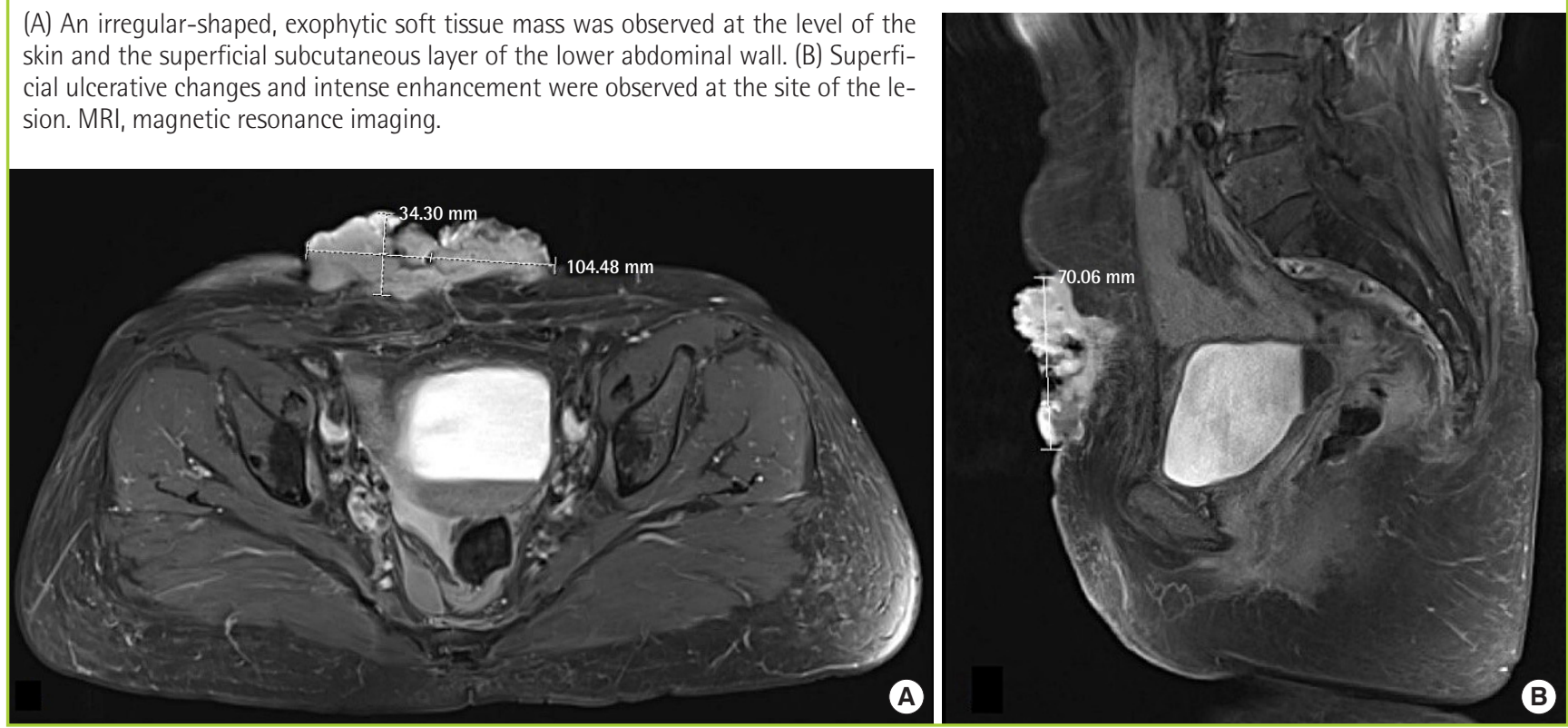

hysterectomy in 1999, with metastasis to the lung, for which she underwent a wedge resection procedure in 2001. Radiation therapy was performed post-hysterectomy in 1999, with regular follow-up. Since the wedge resection procedure in 2001, the patient had not been diagnosed with any tumor other than the abdominal mass. The patient had no personal or family history of skin cancer or other skin diseases. Laboratory studies, including a complete blood cell count, blood chemistry profile, test for sexually transmitted infections, urinalysis, chest X-ray, and electrocardiogram, were negative or within normal limits. ABCC was diagnosed with a skin biopsy, and magnetic resonance imaging confirmed that the ABCC existed only at the level of the skin and the superficial subcutaneous layer of the lower abdomi- nal wall (Fig. 2). The resection margins were determined according to international guidelines $[12,13]$. Intraoperatively, an elliptical wide excision with safety margins of $2.0 \mathrm{~cm}$ was made under general anesthesia (Fig. 3). The wound was closed using an advanced flap technique consisting of the elevation of the cephalic and caudal skin flaps. Complete excision with a negative margin was confirmed by frozen section examination in the operating room, and the final histopathological findings also confirmed the result. As positron emission tomography/computed tomography showed hypermetabolic lymph nodes in both inguinal areas, bilateral inguinal lymph node biopsies were performed. The histopathological examination showed proliferation of basaloid cells extending into the dermis with cyst-like 


\section{Fig. 4. Histopathological examination}

(A) Interweaving cords and varying-sized islands of basal cells, surrounded by a mucinous stroma, were observed (H\&E, $\times 40)$. (B) The center of the structure contained many basaloid cells with dark-staining nuclei and little cytoplasm. The entrapment of the mucinous stroma between the anastomosing strands of cells and within cell islands appeared as gland-like or tubular structures $\left(H \& E_{1} \times 200\right)$.
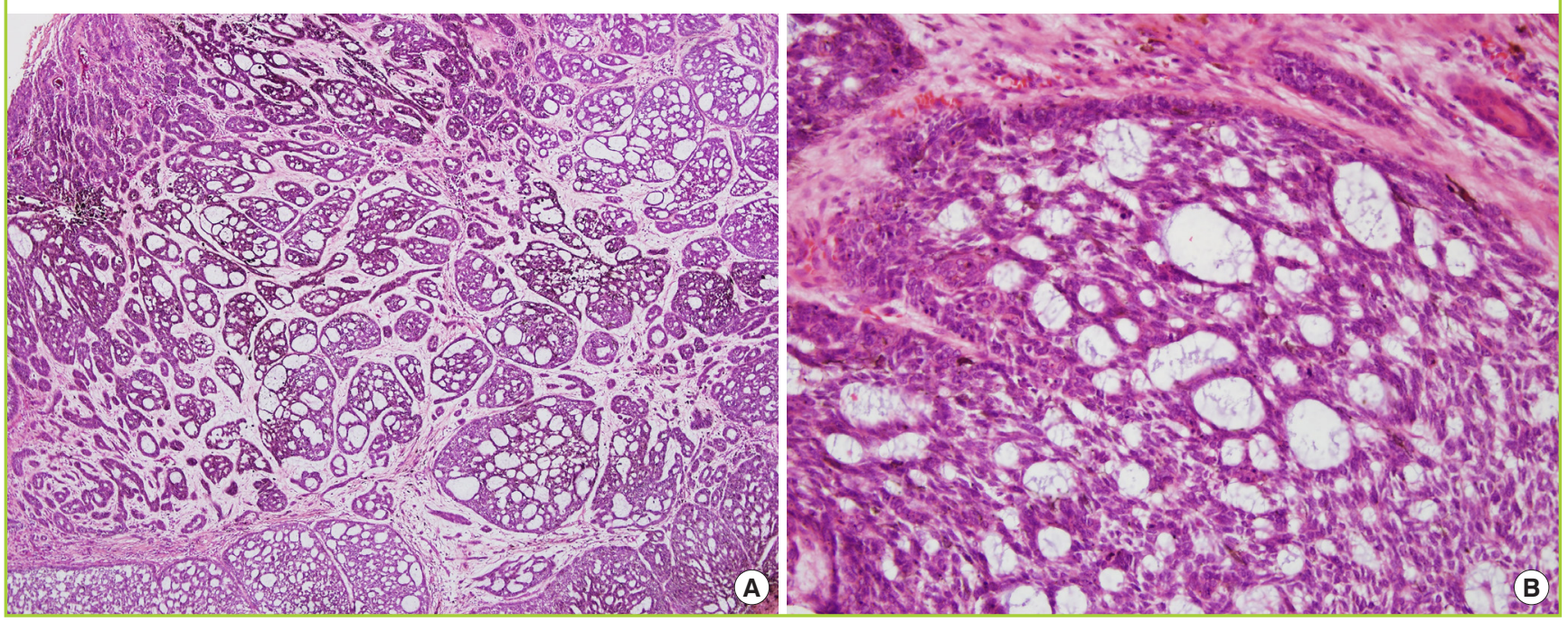

\section{Fig. 5. Postoperative photographs of the patient}

(A) A clinical picture illustrating the patient's immediate postoperative status. (B) Two-month follow-up picture of the patient showing a wellhealed wound, without any complications or signs of recurrence.

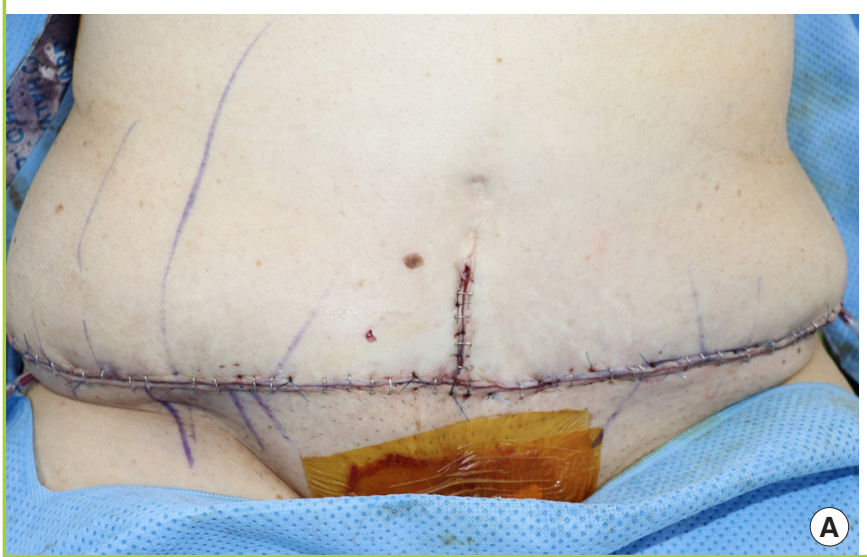

enlarged structures (Fig. 4A). Peripheral palisading was prominent in many of the cellular aggregates. In the center of the structure, many basaloid cells were observed, with dark-staining nuclei and little cytoplasm. The entrapment of the mucinous stroma between the anastomosing strands of cells and within cell islands produced the appearance of gland-like or tubular structures (Fig. 4B). Both the clinical and histopathological features of the carcinoma were consistent with adenoid BCC. There was no lymphovascular invasion within the peripheral or deep resection margin, and the depth of involvement was 1.5 $\mathrm{cm}$. On the immunohistochemical analysis, the atypical cells stained positive for Bcl-2 and c-kit and negative for HMB45, S-100, and epithelial membrane antigen. The surgical wound

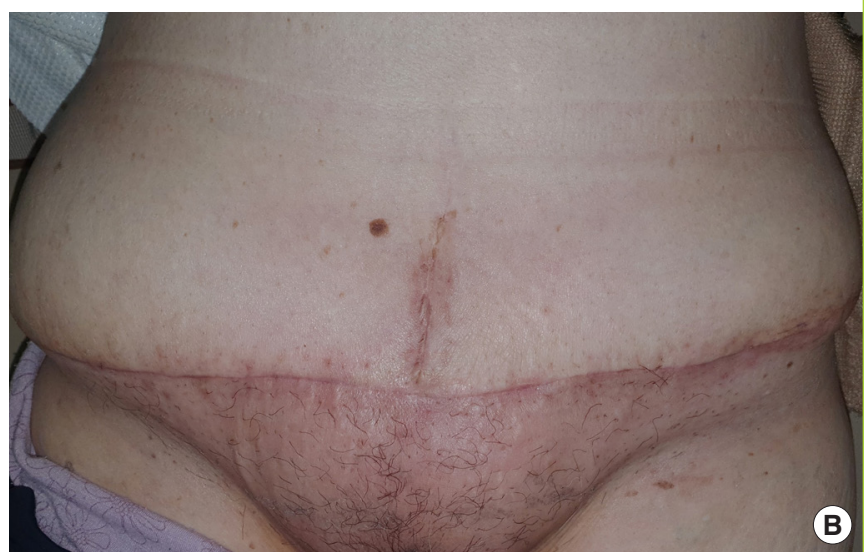

healed well without complications (Fig. 5). Upon consultation with an oncologist, treatment was terminated with surgical resection, and regular follow-up was conducted.

\section{DISCUSSION}

BCC represents approximately $70 \%$ of all malignant diseases of the skin, $75 \%$ of nonmelanoma skin cancers, and $65 \%$ of epithelial tumors [1-3]. A recent Korean study reported that from 1999-2002 to 2011-2014, the incidence rate of BCC increased notably, from 1.18 to 2.35 per 100,000 people in men and from 0.98 to 2.25 per 100,000 people in women [6].

BCCs occur most commonly in sun-exposed areas of the skin, 
such as the face and neck (80\%-90\%); the remaining 10\%-15\% of cases occur in areas not exposed to sunlight, such as the abdomen, armpit, and chest $[4,6]$. The histological diagnosis and classification of $\mathrm{BCC}$ is essential for evaluating the risk of recurrence and comparing treatment outcomes [1-3]. Histologically, BCC can be classified as nodulocystic (35.4\%), mixed (30.1\%), infiltrative (9.3\%), superficial (6.7\%), micronodular (6.2\%), adenoid (5.9\%), metatypical (4.0\%), morpheaform $(2.1 \%)$, or fibroepithelioma (0.3\%) [3-5,14]. Of these, ABCC is a rare histopathological subtype that exhibits tubular differentiation and that has not been reported to occur preferentially at specific sites $[4,5,14]$. Histopathologically, this rare variant of BCC consists of small uniform cells with peripheral nuclear palisading, arranged in nests and cords with intertwining strands $[1,5]$. The nuclei may arrange radially around islands of connective tissue, resulting in a tumor with a lace-like pattern. A colloidal eosinophilic substance or amorphous granular material is sometimes observed within the tubular structure of the lumen. However, studies have not yet determined the precise composition of this material or the exact details of the secretory activity of the lumen-lining cells $[1,5]$.

Although exposure to ultraviolet light is considered the single most important risk factor for BCC, radiation therapy, exposure to arsenic or coal tar derivatives, scars, burn sites, chronic inflammation, ulcers, and immune deficiencies are also associated with an increased risk of BCC $[1,2]$. Radiation therapy for leiomyosarcoma may have been the pertinent risk factor for $\mathrm{BCC}$ in the patient described in the current study. A cohort study conducted in 2011 using data from the U.S. Surveillance, Epidemiology, and End Results cancer registries reported that approximately $8 \%$ of all second solid tumors in adult cancer survivors may be related to the radiation therapy for the original cancer [11]. Evidence of radiation-induced skin cancers has been reported in uranium miners, radium dial painters, radiologists, and patients with a history of using early World War II-era highvoltage cathode-ray tube oscilloscopes, as well as in patients treated with X-rays for childhood acne, tinea capitis, or thymic enlargement $[7,8]$. Possible mechanisms of the development of radiation-associated BCC include radiation-related DNA damage, which induces an intricate range of cellular responses involving the cell signaling pathways implicated in the progression of radiation-induced tumors over time, as well as other factors that may make patients more susceptible to radiation-induced BCCs [9].

Heckmann et al. [15] found that several facial areas, such as the medial quadrant of the orbit, showed high frequencies of BCC despite low ultraviolet light exposure; these areas were found to be characterized by a concave shape, reduced skin ten- sion, and the presence of marked skin folds. Heckmann et al. further suggested that the disturbed cell-matrix interactions at these sites may contribute to the development of BCCs. This aligned with the morphological features of our patient, as her abdomen displayed folds (Fig. 1).

As shown in our case, BCC may occur in sun-protected areas of the body, which can be explained by prior radiotherapy and morphological features of the area. Although clinically, BCCs tend to be relatively stable, occasionally the lesions may grow aggressively and infiltrate the surrounding structures, as in our patient [1-3]. Risk factors for recurrence include the location and size of the tumor, the histological tumor type, and the treatment strategies used. In patients with a history of radiotherapy, lesions that developed longer ago, are located in the center of the face or ear, exceed $2 \mathrm{~cm}$ in size, and are histologically infiltrative, micronodular, and morpheaform have been found to show a higher risk of recurrence $[1,2,4]$. Infiltrative and micronodular variants such as $A B C C$ are more aggressive and are associated with higher recurrence risk $[1,4,5]$.

This report highlights the importance of performing a complete cutaneous examination, including of sun-protected sites, to identify lesions present in areas of the skin that have been irradiated, especially in patients with a history of radiation therapy. Regardless of pigmentation, any small nodular lesion can be a candidate for BCC. Early detection and appropriate treatment can reduce morbidity due to this condition.

\section{NOTES}

\section{Conflict of interest}

No potential conflict of interest relevant to this article was reported.

\section{Ethical approval}

The study was performed in accordance with the principles of the Declaration of Helsinki. Written informed consent was obtained.

\section{Patient consent}

The patient provided written informed consent for the publication and the use of her images.

\section{Author contribution}

Conceptualization: Cheon YW. Data curation: Kim SE. Formal analysis: Kim JH. Funding acquisition: Cheon YW. Methodology: Kim JH. Project administration: Cheon YW. Visualization: Kim SE. Writing - original draft: Kim JH. Writing - review \& editing: Cheon YW. Approval of final manuscript: all authors. 


\section{ORCID}

Ji Hun Kim https://orcid.org/0000-0002-1947-2476

Sun Eung Kim https://orcid.org/0000-0001-6591-4389

Young Woo Cheon https://orcid.org/0000-0003-2940-292X

\section{REFERENCES}

1. Cockerell CJ, Tran KT, Carucci J, et al. Basal cell carcinoma. In: Rigel DS, Robinson JK, Ross M, et al. editors. Cancer of the skin. 2nd ed. Philadelphia: Elsevier Saunders; 2011. p. 99123.

2. Kim DP, Kus KJB, Ruiz E. Basal cell carcinoma review. Hematol Oncol Clin North Am 2019;33:13-24.

3. Koyuncuer A. Histopathological evaluation of non-melanoma skin cancer. World J Surg Oncol 2014;12:159.

4. Kim SH, Ko WT, Suh MK, et al. A case of axillary adenoid basal cell carcinoma. Ann Dermatol 2008;20:22-5.

5. Saxena K, Manohar V, Bhakhar V, et al. Adenoid basal cell carcinoma: a rare facet of basal cell carcinoma. BMJ Case Rep 2016;2016:bcr2015214166.

6. Oh CM, Cho H, Won YJ, et al. Nationwide Trends in the Incidence of Melanoma and Non-melanoma Skin Cancers from 1999 to 2014 in South Korea. Cancer Res Treat 2018; 50:729-37.

7. Chen F, Yang SF, Chen CH, et al. Secondary basal cell carcinoma of scalp after radiotherapy: a case report. Medicine (Baltimore) 2018;97:e12170.
8. Wolfe CM, Green WH, Hatfield HK, et al. Multiple secondary cutaneous tumours following electron beam radiotherapy for cutaneous malignancies of the scalp. Australas J Dermatol 2012;53:233-8.

9. Li C, Athar M. Ionizing radiation exposure and basal cell carcinoma pathogenesis. Radiat Res 2016;185:217-28.

10. Zargari O. Radiation-induced basal cell carcinoma. Dermatol Pract Concept 2015;5:109-12.

11. Berrington de Gonzalez A, Curtis RE, Kry SF, et al. Proportion of second cancers attributable to radiotherapy treatment in adults: a cohort study in the US SEER cancer registries. Lancet Oncol 2011;12:353-60.

12. National Comprehensive Cancer Network (NCCN). NCCN Clinical Practice Guidelines in Oncology [Internet]. Plymouth Meeting, PA: NCCN [cited 2019 Sep 30]. Available from: https://www.nccn.org/professionals/physician_gls/ default.aspx\#nmsc.

13. Bichakjian C, Armstrong A, Baum C, et al. Guidelines of care for the management of basal cell carcinoma. J Am Acad Dermatol 2018;78:540-59.

14. Agarwal A, Raja A, Mahalingam S, et al. Multiple adenoid basal cell carcinoma: an uncommon presentation. Indian J Dermatol Venereol Leprol 2019;85:393-6.

15. Heckmann M, Zogelmeier F, Konz B. Frequency of facial basal cell carcinoma does not correlate with site-specific UV exposure. Arch Dermatol 2002;138:1494-7. 